\title{
Nature, Information \& [Digital] Technology [NIT]: Introduction \& Elements
}

\author{
N.E.Myridis \\ Assistant Professor of Informatics \\ Computer Lab, Aristotle University of Thessaloniki, Greece \\ Tel: 30-2310-996-970Ｅ-mail: myridis@nestoras.ee.auth.gr, nmyridis@theo.auth.gr
}

Received: Novembr 9, 2010

Accepted: March 1, 2011

doi:10.5539/mas.v5n3p119

\begin{abstract}
Modern era is being characterized by the influence of digital and computer systems. These systems dominate the development of sciences, thus leading to a fuzzy distinction between pure scientific innovation and digital-based progress (e.g. DNA sequencing). However, there is a corresponding directional arrow having as an ending point the face of the digital era; the starting point is ever the origin of natural elements. The contents of this presentation are an attempt to trace in depth and then to provide an overview, as a result of a composite process, of the following assemblage of fundamental elements. These elements refer to the multi-faceted thematic realm of: (a) the 'physical world' (the Universe), (b) the information which is usually emitted by this world (though often tacitly), and finally (c) the means and methodology used by modern society in order to deal with this information. The processing of this information is performed, in the vast majority of cases, by means and in terms of digital logic and digital systems. The matter of this presentation should be considered as a generalized introduction to the complete solid of 'Nature, Information and Technology' (the NIT whole).
\end{abstract}

Keywords: Information, Analog \& digital systems, Infrastructure, Informatics, NIT whole

\section{Introduction}

Events encountered during the research for a book (Myridis, 2006) produced a spontaneous fermentation of ideas. In this context, we present in this paper consequent results as a basis for introducing new insight into the chain 'Nature - Information - Technology'.

Thus, digital systems (Latham \& Sassen, 2005) and inherent digital logic could have been viewed with a generic and universal approach. They have therefore been incorporated into the wider context to which they belong, in terms of space and time -i.e. in Cosmos. This reasoning behind the treatment of the themes of this study brought the observation of digital systems to the very starting point of their constituent properties (Grier, 2005), within the context of Universe (creation), i.e. of the unified whole of the material world. Furthermore, the proper redetermination and relocation of the theoretical and mathematical infrastructure of these systems and of their logic was attempted by the use of purely computational methods (Myridis \& Chamzas, 1998). This led to the assignment of the theoretical skeleton and infrastructure of digital expertise to areas of more general thinking. In this way, the legitimacy of digital logic was elevated to the realm of a broader design legitimacy and wisdom in the world.

\section{Cosmos \& Information}

Corresponding scaling and insight, analyses and conclusion were also reached for the other two areas of the subject, i.e. the natural world (the creation) and the information connected to it. An attempt was therefore deemed necessary: an abstract presentation of the foundation and mounting of the structure of the material world on the firm and entirely admirable basis of a variety of (mathematical (Finch, 2005), physical (Gschneidner et al, 2005), chemical etc.) constants (Dunlap, 1998). On the other hand, analyses of fundamental information metrics were also developed. These analyses provide more and stricter criteria and premises in the transaction of information quantification. In parallel- and this may well be more important- they open new inroads into the depth of the world of information (Triantaphyllou \& Felici, 2006), which do, indeed, yield unknown capitals of knowledge to the observer. Finally, within the overall assemblage of a diverse pattern of analyses and conclusions, we can derive important applications, both of a broader interest and also sharply focused, from the domain of digital systems and computers (Tocci et al, 2006), which indicate the vital and unique contribution of these systems to the sequence (Fig.1) 


\section{Cosmos $\Rightarrow$ information $\quad \Rightarrow \quad$ application.}

In the course of the research, epistemological features surface, and their presentation is deemed both inevitable and crucial. At every point investigated in this work, we are faced with clear and wisely-perceived perfection, causality and legitimacy (Barrow \& Tipler, 1986).

\section{Systems \& Information}

The treatment of the subject matter is carried out by its tacit division mainly into the following two thematic areas:

(a) analog \& digital infrastructure, and

(b) information.

We can therefore observe two corresponding parts of the work, where each of the previous components forms the kernel and the directional axis for the study and research. In both parts, there is a development of the argument, which places each of the components as a structural or embedded core into a wider totality. The latter is then accounted for as part of whole of creation. This treatment yields, as its most important result, a unified view and consideration of the corresponding two thematic areas, within the universal context of the world to which they belong. However, there are horizons remaining unseen because of extremely high focus resolution. We shall take a wider view in order to reveal and evaluate lost details and unseen horizons. These details have by and large been usually lost due to the lack of inter-thematic correlation of subjects related to Technology with subjects in adjacent or remote fields.

\section{Principles}

In the first part, the thematic constituent is analog and digital infrastructure. Thus we attempt to characterize this infrastructure by evaluation and to correlate it by perception to the wider fields of science and creation. In this part, principal terms such as those of 'digital', 'analog', 'harmony', 'symmetry' etc. (Muller, 1998) are considered, developed and cross-correlated. There is an analysis and presentation of the way creation is structured from its constituent parts (properties, infrastructure (Mysen \& Richet, 2005) etc.) and from this analysis the fundamental relationship between digital systems and the natural world is made clear. Constants (mathematical, physical etc.) have critical and vital value for this infrastructure. The whole Universe has been constructed on the structure of constants; extensive reference should therefore be made to them. Particular weight is also carried by the development of certain principles, which, though unseen, discreetly permeate the realm and functionality of creation. Such principles are, for example, the friendliness of technology (Note 1), inherent order, adaptation to orderliness etc. Equally important are the fundamental principles of science which are well-defined in this context. The relative principles occupy a dominant position in modern human life, as they determine, with the necessary accuracy, the limits of science, as well our attitude towards it. The collation of these principles, their definition and their formulation is a prime contribution of such a work (Myridis, 2006) The following arise in an entirely natural way from this expansion of our study to the limits of science:

(a) the extreme boundaries of science

(b) the specific regions of its scope.

\section{Digital Information Systems in Cosmos}

Modern digital technology and Computer Science are descendants of the creation (since they have borrowed from it the material elements for their structure) and of the human spirit and civilization (Kahn, 2010). Through feedback and reinforcement, these filial expressions of civilization serve a useful purpose to people and to creation:

Feedback: because they contribute to the decoding and understanding of the facets of creation to an ever greater extent.

Reinforcement: because they allow for the exploitation of the latent potential within creation, for the improvement of human life. They also permit us to reconsider our attitude to and perception of the world and things in it, as knowledge becomes more qualitative and extensive.

\section{Information and Informatics}

In the second part, we consider and use the term and quantity of information as the core. The word 'information' gives the name to the term 'Informatics' (Gammack et al, 2007). This connotation is also -necessarily- seen within the broader context of creation; indeed information is a quantity which is to be found throughout the 
whole of creation (Tartar, 2006). It is the main support on which the all the physical processes and observation of them are based. In this part, original and fundamental conclusions are drawn, which make a genuine contribution to the in-depth analysis of Information Theory (Cover \& Thomas, 1991). Information itself, thanks to its global distribution, is essentially the 'algorithm of creation'. Moreover, we all receive this information on a daily basis, as we participate in the same world, though we do not evaluate or develop it to any great extent. Each person therefore may belong to one of the following categories:

(a) In first category, someone receives information by the sensory organs, but he cannot deeply understand or evaluate it due to the lack of sufficient knowledge. Everyone belongs in part to this class, as the largest quantity of knowledge remains unknown. As an example, we might cite the case of the particle form and composition of materials, which was unknown some years ago. The reason for our inability to perceive and see the particle synthesis of Nature results from the inability of human sensors (eyes). Modern technology and Informatics strive to overcome such defects (Iskander et al, 2004). Technology nowadays is almost exclusively based on Computer Science (International Chicken Genome Sequencing Consortium, 2004). It supplies humanity with the means and tools for investigation of the mystery of microcosm (Jureller et al, 2006) (m-cosmos) and Macrocosm (Kallenrode, 2001), (Sky \& Telescope, 2006) (M-cosmos), as well as of the human body, too. Computer Science undertakes the work of analyzing, evaluating and developing the products of this investigation, i.e. the hidden information. The relationship, between information and Informatics results from this.

(b) The second category refers to the case where people possess sufficient knowledge of the various subjects of creation as well as of their life, e.g. the particle nature of materials, quantum states (Vogel, 2001), wave theory of light (Einstein, 20057) etc. However, they do no more than record and reproduce this knowledge, without evaluating and developing it in a reductive process. They cannot, or rather, do not desire to observe and process information through those filters, which are able to explain the structural identities of the information, i.e. causality (Papoulis et al, 2002), legitimacy and teleology. They therefore receive the information, analyze and process it and, in the end, reach important conclusions and a reasonable amount of knowledge concerning it. They recognize and admire the inherent wisdom, perfection and actuality of things. Often they actually foresee this. However, they do not proceed to higher levels of investigation, towards the discovery of the purpose of perfection. Since all things and beings usually serve specific purposes (e.g. the well-known Anthropic Principle) (Cirkovic, 2004).

(c) The last category refers to the highest level of hierarchy. We are, in essence, called upon to elevate the level of the observation and processing of information; not only to possess and record the inherent knowledge of things we have acquired, but much more to interpret it with proper reference to causality, employing two excellent tools for the purpose: logic and experience.

\section{Conclusion}

The analyses in the second part reveal some unknown elements, which may function as sufficient criteria for the study and characterisation of physical and discrete phenomena.

As a conclusion, we believe that the unified view of the solid 'Nature, Information \& Technology' which has been introduced herein, will inaugurate a new turn in the concept of modern sciences, while giving amazing and fruitful results.

\section{References}

Barrow J.D. \& Tipler F. (1986). The Anthropic Cosmological Principle. N.York: Oxford University Press.

Cirkovic, M.M. (2004). The Anthropic Principle and the duration of the Cosmological Past. Astronomical \& Astrophysical Transaction, vol.. 23, p.567-597.

Cover, T.M. \& Thomas, J.A. (1991). Elements of Information Theory. N.York: J.Wiley.

Dunlap R.A. (1998). The Golden Ratio \& Fibonacci Numbers. Singapore: World Scientific Publications.

Einstein, A. (2005). The meaning of relativity $\left(7^{\text {th }}\right.$ ed.). Princeton: Princeton University Press.

Finch, S.R. (2003). Mathematical constants. Cambridge: Cambridge University Press.

Gammack, J., Hobbs, V., Pigott, D. (2007). The book of Informatics. S.Melbourne: Thomson.

Grier, D.A. (2005). When computers were human. N.Jersey: Princeton University Press.

Gschneidner, K.A., Bunzli, J-C. \& Pecharsky, V.K. (eds.). (2005). Handbook on the Physics and Chemistry of Rare Earths. Amsterdam: Elsevier. 
International Chicken Genome Sequencing Consortium. (2004). Sequence \& comparative analysis of the chicken genome provide unique perspectives on vertebrate evolution, Nature, vol. 432, p.695-716.

Iskander, D.R., Collins, M.J., Mioschek, S., Trunk, M. (2004). Automatic Pupillometry from Digital Images. IEEE Trans.Biomedical Engineering vol.51(9), p.1619-1627.

Jureller, J., Kim, H., Scherer, N. (2006). Stochastic Scanning Multiphoton Multifocal Microscopy. Optics Express, vol.14(8), p.3406-3414.

Kahn, P. (2010). Technological Nature. (Adaptation and the Future of Human Life). Boston: MIT Press

Kallenrode, M-B. (2001) Space Physics, Berlin: Springer.

Latham R. \& Sassen S. (eds.) (2005). Digital Formations. N.Jersey: Princeton University Press.

Muller, C. (1998). Analysis of spherical symmetries in Euclidean spaces. Springer.

Myridis N.E., (2006). The Information of Observation: From Analog to Digital. Thessaloniki: Kyriakides Pbls (in Greek).

Myridis, N.E. \& Chamzas, C. (1998). Sampling on Concentric Circles. IEEE Trans. Medical Imaging, vol.17(2), p.294-299.

Mysen, B. \& Richet, P. (2005). Silicate Glasses and Melts, Amsterdam: Elsevier.

Papoulis, A. et al., (2002). Probabilities, Random Variables \& Stochastic Processes, N.York: McGraw-Hill.

Sky \& Telescope's Beautiful Universe, (2006 edition).

Tartar, L. (2006). An Introduction to Navier-Stokes Equation and Oceanography. The Netherlands: Springer.

Tocci, R., Widmer, N., Moss, G. (2006). Digital Systems: Principles \& Applications. N.Jersey: Prentice Hall.

Triantaphyllou, E. \& Felici, G., (2006). Data Mining \& Knowledge Discovery approaches based on rule induction techniques. N.York: Springer.

Vogel, W., Welsch, D-G. \& Wallentowitz, S. (2001). Quantum Optics. An Introduction. Berlin: Wiley-VCH.

\section{Notes}

Note 1. The principle of the friendliness of technology determines if a technological achievement is friendly to human beings or not.

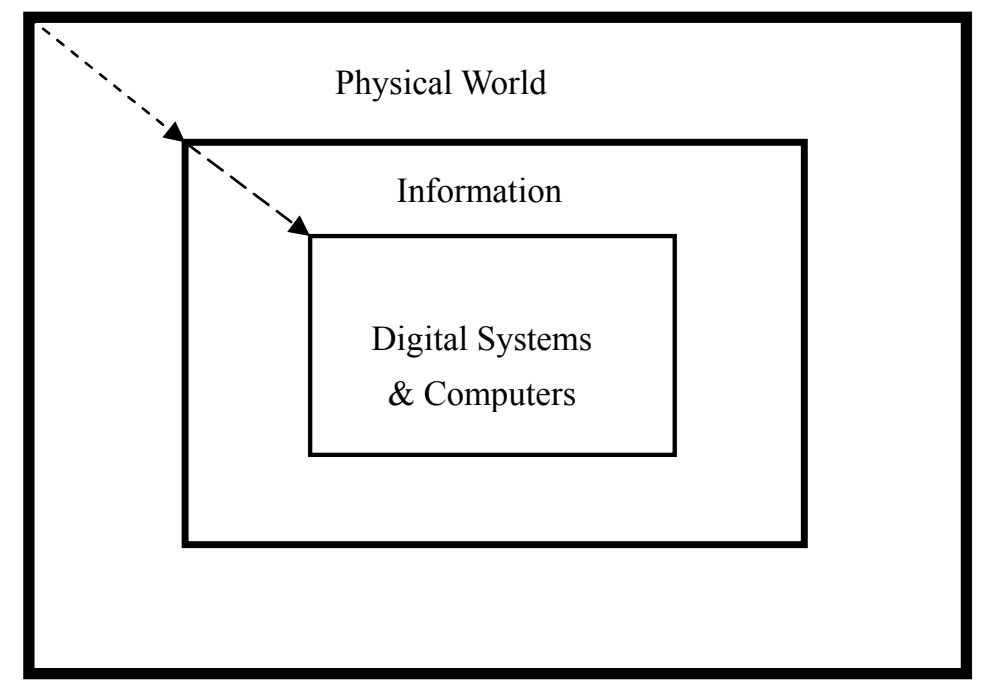

Figure 1. The stratification of the NIT whole 